\title{
Metformin increases sensitivity of osteosarcoma stem cells to cisplatin by inhibiting expression of PKM2
}

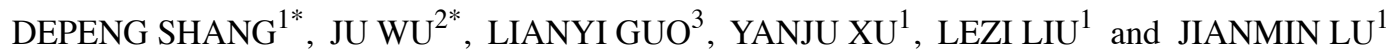 \\ Departments of ${ }^{1}$ Orthopedics, ${ }^{2}$ General Surgery, Zhongshan Hospital Affiliated of Dalian University, Dalian, \\ Liaoning 116001; ${ }^{3}$ Department of Gastroenterology, The First Affiliated Hospital of \\ Jinzhou Medical University, Jinzhou, Liaoning 121001, P.R. China
}

Received February 17, 2017; Accepted March 20, 2017

DOI: $10.3892 /$ ijo.2017.3950

\begin{abstract}
Multiple drug resistance is reported to be a major obstacle in treatment of osteosarcoma (OS). Research has demonstrated that small subsets of cells called cancer stem cells (CSCs) are responsible for multiple drug resistance. CSCs are potential targets for reversing chemoresistance. In the present study, we compared cisplatin sensitivity between OS stem cells and OS non-stem cells. We confirmed that OS stem cells showed significant cisplatin-resistance compared with the OS non-CSCs. Mechanically, we proved that overexpression of the pyruvate kinase isoenzyme M2 (PKM2) was responsible for the resistance to cisplatin in OS stem cells. As a potential strategy, we found that co-treatment with metformin significantly decreased the half maximal inhibitory concentration $\left(\mathrm{IC}_{50}\right)$ of cisplatin to HOS OS stem cells by downregulating the expression of PKM2. PKM2 downregulation resulted in, metformin inhibited glucose uptake, lactate production and ATP production in HOS CSCs. Therefore, metformin impaired the resistance of HOS CSCs to cisplatin and promoted cisplatin-induced apoptosis. In addition, antitumor effects of other chemotherapeutic drugs such as doxorubicin and 5-fluorouracil were proved to be enhanced by metformin on OS stem cells.
\end{abstract}

\section{Introduction}

Osteosarcoma (OS) is a malignant bone cancer commonly diagnosed in children and adolescents (1). At present, chemotherapy is still an effective treatment to kill OS cells and prolong the life of patients. However, repeated use of chemotherapeutic drugs induces inevitable multiple drug resistance

Correspondence to: Dr Jianmin Lu, Department of Orthopedics, Zhongshan Hospital Affiliated of Dalian University, 6 Jiefang Street, Dalian, Liaoning 116001, P.R. China

E-mail: jnxuechengcao@yeah.net

${ }^{*}$ Contributed equally

Key words: osteosarcoma stem cells, metformin, pyruvate kinase isoenzyme M2, cisplatin resistance, ATP in cancer cells $(2,3)$. Reliable strategies are urgently required to reverse the multiple drug resistance of OS.

Cancer stem cells (CSCs) are small cell subsets existing in cancer. Characteristics of CSCs include high self-renewal capacity and multilineage differentiation potential. They are important for tumor formation and development $(4,5)$. CSCs are considered to be responsible for induction of acquired drug resistance. Continuous chemotherapy induces stemness in OS cells (6-8). CD133, a glycoprotein on cell surface, is identified as the molecular marker of cancer stem cells in many tumors including OS $(9,10)$. CD133-positive OS cells are considered as effective targets for reducing chemoresistance (11).

Metformin is an anti-diabetic drug, which is the first-choice for patients with type-II diabetes (12). Of note, metformin therapy is shown to reduce cancer incidence in patient with diabetes. Metformin treatment reduces the risk of many tumors including breast cancer, lung cancer and colorectal cancers (13-16). However, the mechanism by which metformin inhibits malignancies still remains unclear. Some studies demonstrate that metformin can reduce the production of ATP in cancer cells, therefore inhibiting cell cycle and proliferation of cancer $(17,18)$. In this study, we investigated the potential role of metformin in OS stem cells. We report that metformin treatment can inhibit glucose metabolism and increase sensitivity of OS stem cells to chemotherapy.

\section{Materials and methods}

Cell culture. Human osteosarcoma cell lines HOS, Saos-2 and MG-63 were purchased from American Type Culture Collection (ATCC, Rockville, MD, USA). For separation of cancer stem cells from OS cell lines, HOS, Saos-2 and MG-63 cells were incubated with fluorescein isothiocyanate (FITC) conjugated CD133 antibody (Miltenyi Biotec, Bergisch Gladbach, Germany) for $20 \mathrm{~min}$ at room temperature. Then, cells were washed with cold PBS twice followed by sorting the CD133-positive OS cells and CD133-negative OS cells on a FACS vantage (FACSCalibur, BD Biosciences, Franklin Lakes, NJ, USA). CD133-positive OS cells were considered as the OS stem cells, whereas the CD133-negative OS cells were identified as the OS non-stem cells (9). All of these cells were cultured in DMEM medium supplemented with $10 \%$ fetal bovine serum (FBS, Gibco-Invitrogen, Carlsbad, CA, USA). 
Quantitative real-time polymerase chain reaction ( $q R T-P C R)$. Total RNAs were extracted from HOS, Saos-2 and MG-63 OS cells using Trizol reagent (Invitrogen). cDNA of these cells was synthesized using M-MLV Reverse Transcriptase (Invitrogen) by taking total RNAs as templates. For detecting the expression of PKM2, Real-time PCR was performed using SYBR Premix Ex Taq (Takara, Shiga, Japan) on ABI PRISM 7900 sequence detection system (Applied Biosystems, Carlsbad, CA, USA). GAPDH gene was used as the internal reference to determine the relative expression of PKM2.

PKM2 plasmid and siRNA. To investigate the role of PKM2 in OS stem cells, we overexpressed or knocked down the PKM2 by transfecting with PKM2 plasmid or small interfering RNA (siRNA), respectively. For conducting PKM2 eukaryotic expression plasmid, open reading frame of PKM2 gene was amplified by PCR followed by linking the PCR products to pcDNA3.1 plasmid (Invitrogen). For knockdown of PKM2, siRNA targets to PKM2 was purchased from Genechem Co., Ltd. (Shanghai, China). For transfection, $2 \mu \mathrm{g} / \mathrm{ml}$ PKM2 plasmid or $50 \mathrm{pmol} / \mathrm{ml}$ PKM2 siRNA were transfected into the OS stem cells by using Lipofectamine 2000 (Invitrogen).

Cell viability and $\mathrm{IC}_{50}$. OS cells $\left(1 \times 10^{4}\right)$ were seeded in 96-well plates and cultured at $37^{\circ} \mathrm{C}$. Cells were transfected with PKM2 vector or PKM2 siRNA. At $24 \mathrm{~h}$ after transfection, the cells were treated with $2 \mathrm{mM}$ metformin and $8 \mu \mathrm{M}$ cisplatin for $48 \mathrm{~h}$, cell viability was measured by Cell Counting Kit- 8 (CCK-8, Dojindo, Kumamoto, Japan) assays. The absorbance was measured at $450 \mathrm{~nm}$ by using an ELISA microplate reader (Sunrise microplate reader; Tecan, Mannedorf, Switzerland). Half maximal inhibitory concentration $\left(\mathrm{IC}_{50}\right)$ was calculated according to the results of CCK-8 assays.

Western blot analysis. Total proteins were extracted from OS stem cells and OS non-CSCs using radio immunoprecipitation assay (RIPA) lysis buffer (Cell Signaling Technology, Inc., Danvers, MA, USA). The proteins in cell lysates were separated by $12 \%$ sodium dodecyl sulfate-polyacrylamide gel electrophoresis (SDS-PAGE). Proteins on the gels were then transferred to polyvinylidene fluoride (PVDF) membranes (Millipore, Billerica, MA, USA). The membranes were blocked by $5 \%$ non-fat dry milk for $2 \mathrm{~h}$ followed by incubating with primary antibodies against PKM2, pro-caspase-9, procaspase-3, cleaved caspase-9, cleaved caspase-3 and $\beta$-actin (Cell Signaling Technology, Inc.) overnight. Then, the proteins on PVDF were incubated with appropriate HRP-conjugated secondary antibodies followed by detecting the protein signals by using an enhanced chemiluminescent substrate (Thermo Fisher Scientific, Inc., Waltham, MA, USA).

Analysis of apoptosis. HOS CSCs were transfected with PKM2 vector followed by treating with $2 \mathrm{mM}$ metformin and $8 \mu \mathrm{M}$ cisplatin. The cells were stained using the Annexin V-FITC apoptosis detection kit (Sigma-Aldrich, St. Louis, MO, USA) and subsequently analyzed using flow cytometry (BectonDickinson, San Jose, CA, USA).

Proliferation assay. HOS CSCs were transfected with PKM2 vector followed by treating with $2 \mathrm{mM}$ metformin and $8 \mu \mathrm{M}$ cisplatin. Proliferation of HOS CSCs was measured by using ${ }^{3} \mathrm{H}$-thymidine incorporation assay.

Glucose uptake measurement. HOS CSCs were transfected with PKM2 vector followed by treating with $2 \mathrm{mM}$ metformin and $8 \mu \mathrm{M}$ cisplatin. Relative glucose uptake was measured using an Amplex Red Glucose/Glucose Oxidase assay kit (Molecular Probes, Camarillo, CA, USA) according to the manufacturer's instructions.

Detection of lactate production. HOS CSCs were transfected with PKM2 vector followed by treating with $2 \mathrm{mM}$ metformin and $8 \mu \mathrm{M}$ cisplatin. Production of lactate in HOS CSCs was detected by using a Lactate assay kit (BioVision, Milpitas, CA, USA) according to the manufacturer's protocol.

ATP measurement. HOS CSCs were transfected with PKM2 vector followed by treating with $2 \mathrm{mM}$ metformin and $8 \mu \mathrm{M}$ cisplatin. After treatment, cells were harvested and washed with PBS twice. NP40 solution (1\%) was used to split the cells. ATP in the cell lysates were measured by using ATP Colorimetric/Fluorometric Assay kit (BioVision).

Tumor growth in nude mice. Four-week-old female immunodefcient nude BALB/c mice were purchased from Shanghai Super-B\&K Laboratory Animal Corp., Ltd. (Shanghai, China). For xenografts, mice were injected subcutaneously into the right armpit with $5 \times 10^{6}$ HOS CSCs. Cisplatin $(5 \mathrm{mg} / \mathrm{kg})$ and metformin $(200 \mathrm{mg} / \mathrm{kg})$ were administered by intraperitoneal injection twice a week. Tumor size was measured every five days. Tumor volume was calculated according to the following formula: volume $(\mathrm{V})=1 / 2 \mathrm{x}$ length $\mathrm{x}$ width $^{2}$. For detection of PKM2 expression in tumor tissues, collagenase type III (Worthington Biochemical Corp., Lakewood, NJ, USA) was used to digest the tissues. The animal care and experimental protocols were approved by the Animal Care Committee of The Affiliated Zhongshan Hospital of Dalian University and complied with the recommendations of the Chinese guidelines for the care and use of laboratory animals. The study was approved by ethics committee of The Affiliated Zhongshan Hospital of Dalian University.

Statistical analysis. Data are presented as mean \pm SD and analyzed by using SPSS 14.0. Two-tail Student's t-test and ANOVA were performed to determine the differences. A value of $\mathrm{P}<0.05$ was considered to indicate a statistically significant difference. All the experiments were independently repeated at least 3 times.

\section{Results}

Osteosarcoma stem cells are resistant to cisplatin treatment. To investigate the cisplatin sensitivity of OS stem cells and OS non-stem cells, we first separated them from the HOS, Saos-2 and MG-63 OS cell lines. As shown in Fig. 1A, the separation efficiency was evaluated by flow cytometry. CD133-positive OS cells were considered as the OS stem cells. Whereas, the CD133-negative OS cells were identified as the OS non-stem cells. Results of CCK- 8 assays showed that the sensitivity of OS stem cells (HOS CSCs, Saos-2 CSCs and MG-63 CSCs) 
A
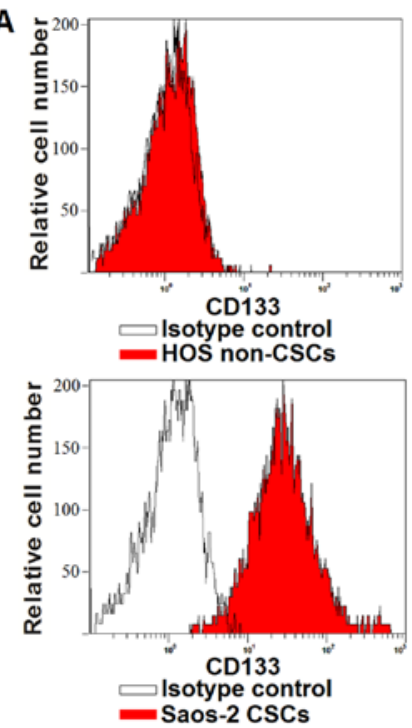

B

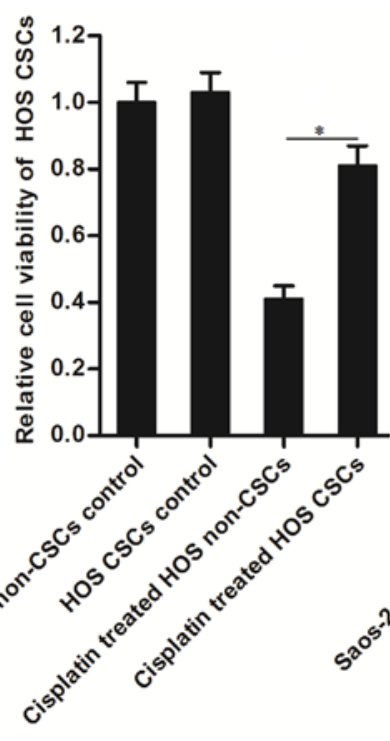

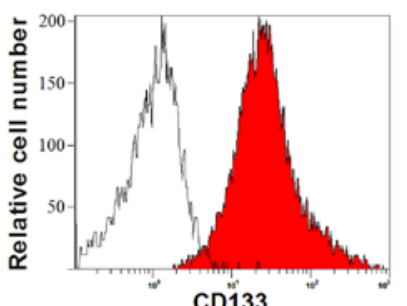

CD133

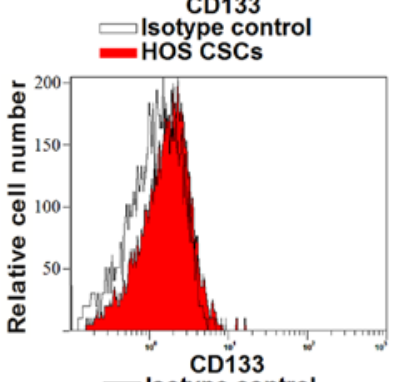

Isotype control

MG-63 non-CSCs

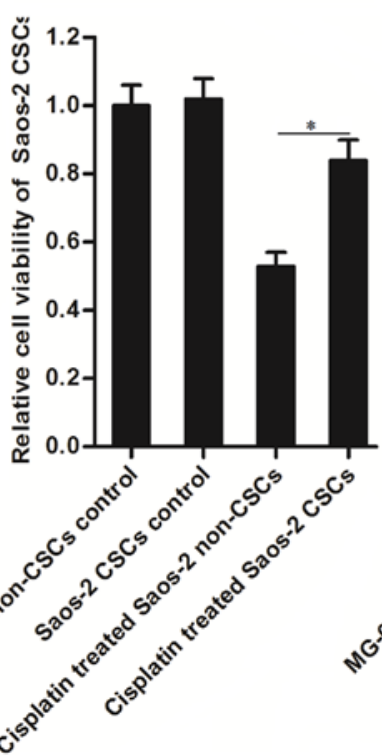

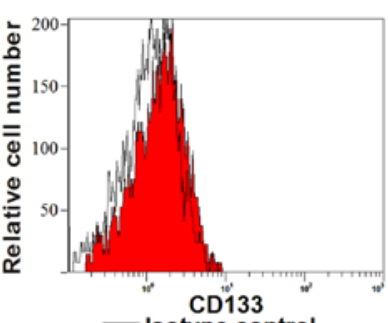

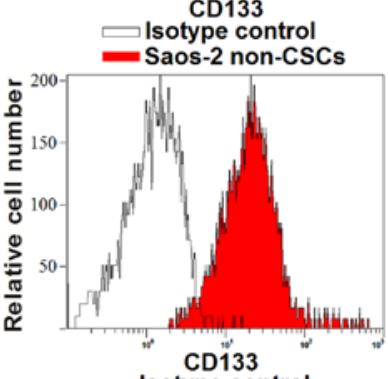

Isotype control

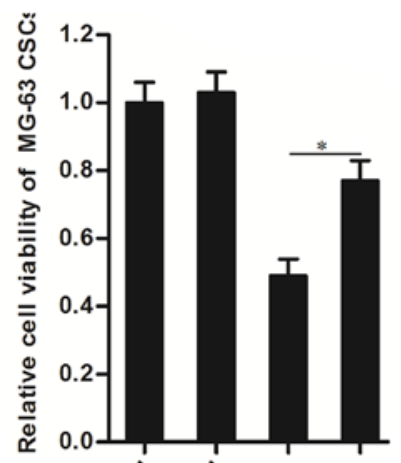

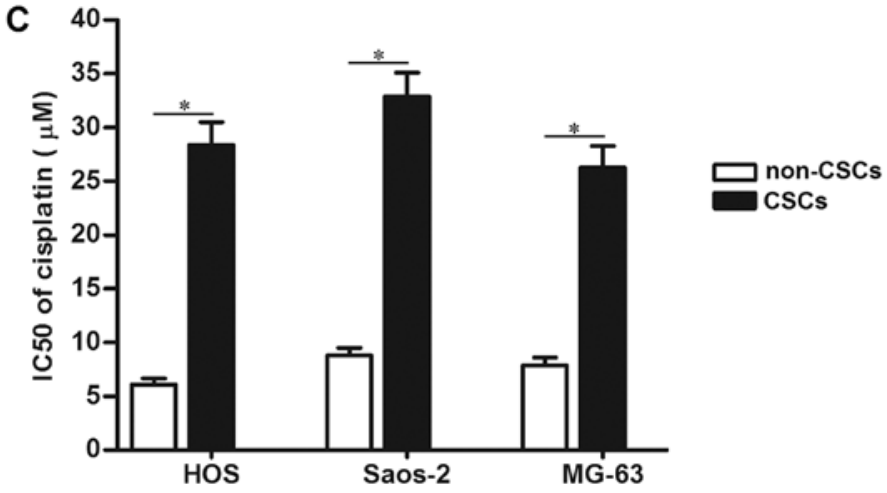

Figure 1. Osteosarcoma stem cells are resistant to cisplatin treatment. (A) HOS, Saos-2 and MG-63 osteosarcoma (OS) cells were incubated with CD133 antibodies followed by sorting the cancer stem cells (CSCs) and non-CSCs on flow cytometry. (B) HOS, Saos-2 and MG-63 CSCs and non-CSCs were treated with $8 \mu \mathrm{M}$ cisplatin for $48 \mathrm{~h}$. Relative cell viability was measured by CCK-8 assays. ${ }^{*} \mathrm{P}<0.05$. (C) $\mathrm{IC}_{50}$ of cisplatin to OS stem cells and OS non-stem cells was determined according to the results of CCK-8 assays. ${ }^{*} \mathrm{P}<0.05$.

to cisplatin was significantly lower than the OS non-stem cells (HOS non-CSCs, Saos-2 non-CSCs and MG-63 non-CSCs) (Fig. 1B). $\mathrm{IC}_{50}$ of cisplatin to OS CSCs (28.4 $\mu \mathrm{M}$ to HOS CSCs, $32.9 \mu \mathrm{M}$ to Saos-2 CSCs and $26.3 \mu \mathrm{M}$ to MG-63 CSCs) was significantly higher than their corresponding OS non-CSCs (6.1 $\mu \mathrm{M}$ to HOS non-CSCs, $8.8 \mu \mathrm{M}$ to Saos-2 non-CSCs and
$7.9 \mu \mathrm{M}$ to MG-63 non-CSCs) (Fig. 1C). Taken together, we demonstrated that osteosarcoma stem cells showed resistance to cisplatin treatment.

Cisplatin-resistance in OS stem cells is dependent on the overexpression of PKM2. PKM2 plays an important role in 
A
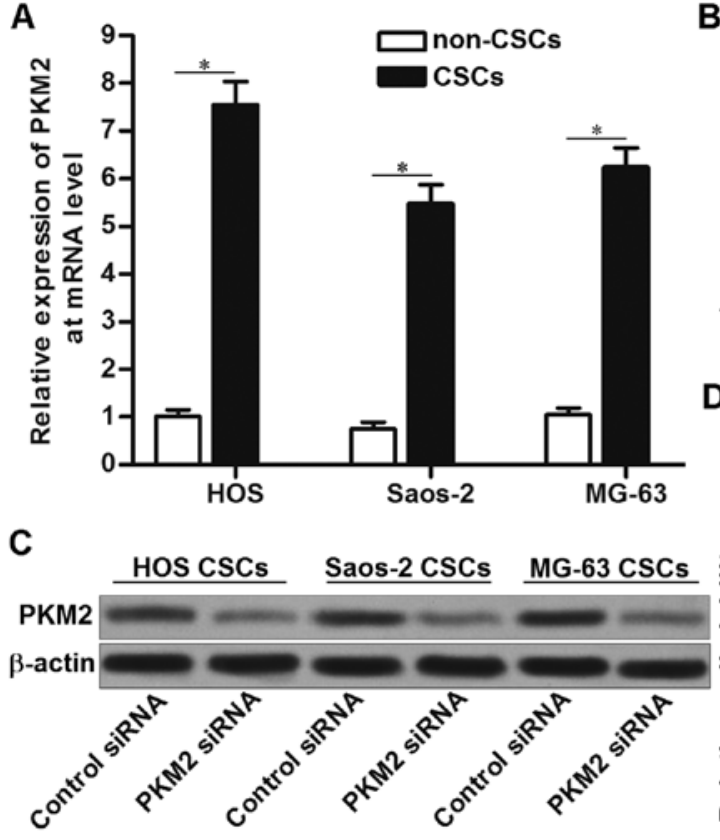

B
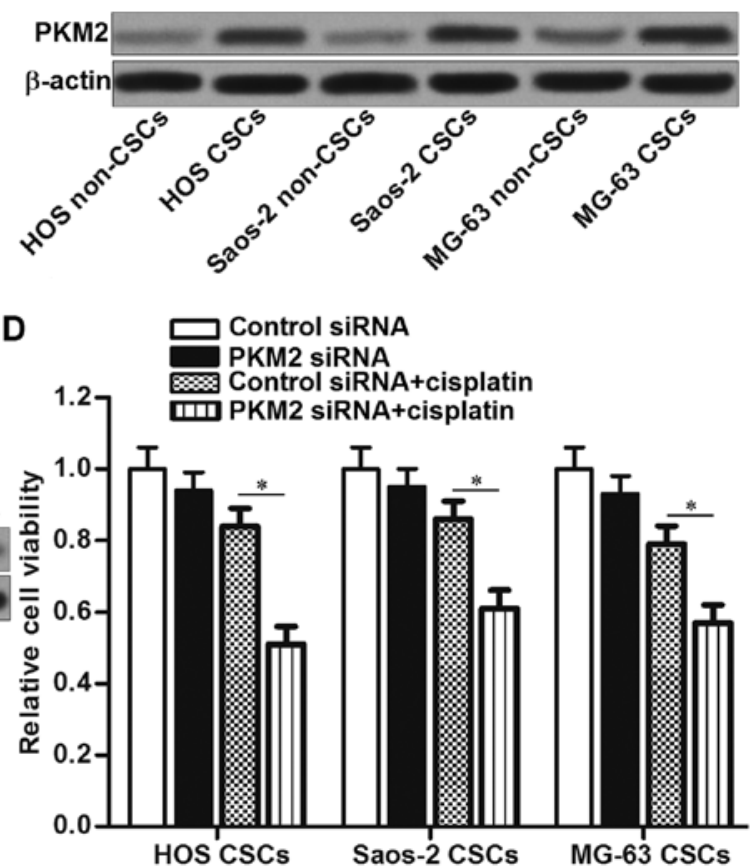

Figure 2. Role of PKM2 in cisplatin-resistance in osteosarcoma (OS) stem cells. (A) mRNA expression of PKM2 in HOS, Saos-2 and MG-63 cancer stem cells (CSCs) and their corresponding non-CSCs was detected by qRT-PCR analysis. " P<0.05. (B) Protein expression of PKM2 in HOS, Saos-2 and MG-63 CSCs and their corresponding non-CSCs was detected by western blot analysis. (C) Expression of PKM2 in HOS, Saos-2 and MG-63 CSCs was knocked down by PKM2 specific siRNA. Western blot analysis was performed to detect the transfection efficiency of PKM2 siRNA. (D) PKM2 siRNA increased the sensitivity of HOS, Saos- 2 and MG-63 CSCs to cisplatin $(8 \mu \mathrm{M})$ treatment. Cell viability was measured by CCK-8 assays. "P<0.05.

tumor growth, development and chemoresistance $(19,20)$. In our study, we found that the expression of PKM2 was significantly overexpressed in OS stem cells compared with that in their corresponding OS non-stem cells at mRNA (Fig. 2A) and protein levels (Fig. 2B). To investigate the role of the overexpressed PKM2, we transfected the HOS, Saos-2 and MG-63 CSCs with PKM2 siRNA. Effects of PKM2 siRNA on OS stem cells are shown in Fig. 2C. Noteworthy, we observed that knockdown of PKM2 by the specific siRNA restored the sensitivity of OS stem cells to cisplatin treatment (Fig. 2D). These results emphasized the key role of PKM2 in cisplatinresistance in OS stem cells.

Metformin resensitizes HOS CSCs to cisplatin by downregulating the expression of PKM2 in vitro. To investigate the potential role of metformin in the sensitivity of OS stem cells to cisplatin, we treated the HOS CSCs and non-CSCs with $8 \mu \mathrm{M}$ cisplatin and $2 \mathrm{mM}$ metformin for evaluating their synergistic effects. We found that metformin markedly enhanced the cytotoxicity of cisplatin to HOS CSCs, whereas the effect of metformin was slight on enhancing the cytotoxicity of cisplatin on HOS non-CSCs (Fig. 3A). Combination with metformin decreased the $\mathrm{IC}_{50}$ of cisplatin to HOS CSCs by $74.9 \%$. In contrast, the reduction of cisplatin $\mathrm{IC}_{50}$ to $\mathrm{HOS}$ non-CSCs was $31.2 \%$ (Fig. 3B). These data demonstrated that metformin was able to enhance the antitumor effect of cisplatin, and HOS CSCs were more sensitive to metforminpromoted cell death than their corresponding HOS non-CSCs. Mechanically, results of western blot analysis showed that metformin treatment significantly downregulated the expres- sion of PKM2 in HOS CSCs (Fig. 3C). We therefore inferred that synergistic effect of metformin on cisplatin-induced cell death was dependent on the inhibition of PKM2. To prove this inference, we transfected the HOS CSCs with PKM2 expression vector. We found that enforced expression of PKM2 'rescued' the HOS CSCs from the combination treatment with cisplatin and metformin (Fig. 3D). These results indicated that metformin had the ability to resensitize OS stem cells to cisplatin by targeting PKM2.

Metformin resensitizes OS CSCs to cisplatin in vivo. To investigate whether metformin enhanced the antitumor effect of cisplatin on OS CSCs in vivo, we established the in vivo model of OS by using HOS CSCs. We observed that single administration with cisplatin slightly reduced the tumor sizes in mice. Of note, although metformin single treatment did not reduce the tumor volume obviously, it strongly enhanced the antitumor effect of cisplatin on HOS CSC model (Fig. 4A and B). After analyzing the expression of PKM2 in tumor tissues formatted by HOS CSCs, we found that metformin significant decreased the expression of PKM2 in HOS CSC in vivo model (Fig. 4C). These results suggested that metformin have to the ability to sensitize the OS CSCs to cisplatin by inhibiting the expression of PKM2 in vivo.

Metformin weakens the glucose metabolism. As PKM2 is a key regulator of glucose metabolism in tumor cells (21), we next studied the effect of metformin on glucose metabolism in HOS CSCs. As shown in Fig. 5A and B, cisplatin treatment did not obviously influence the glucose metabolism in HOS 
A

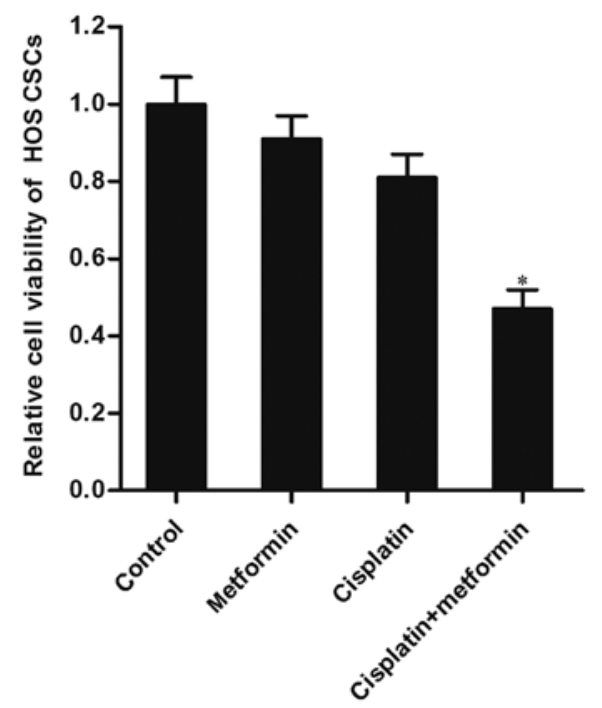

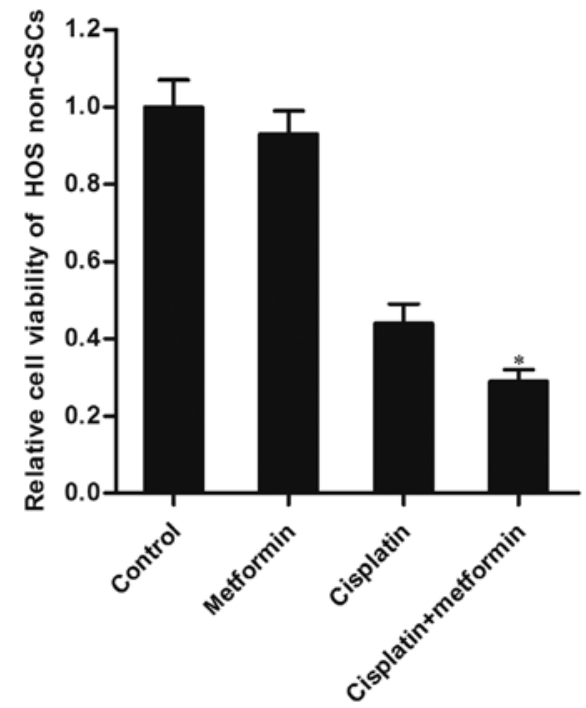

B

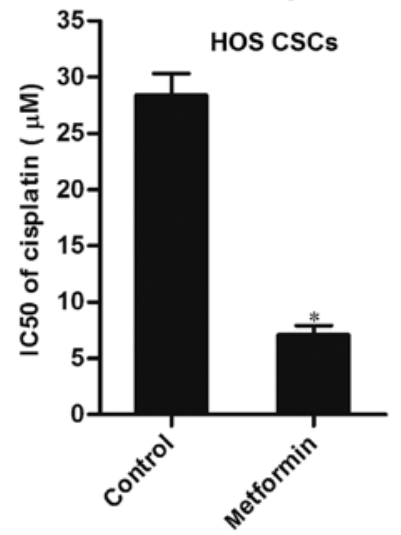

C

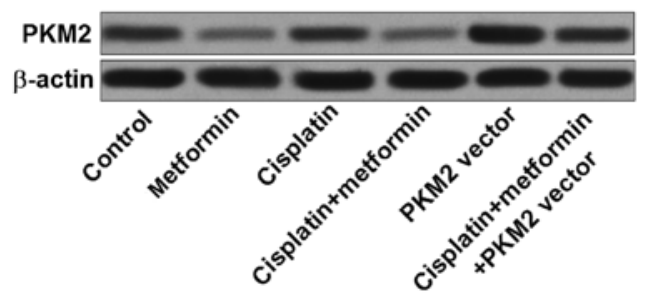

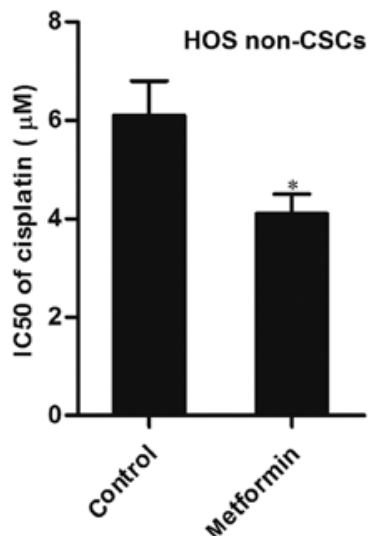

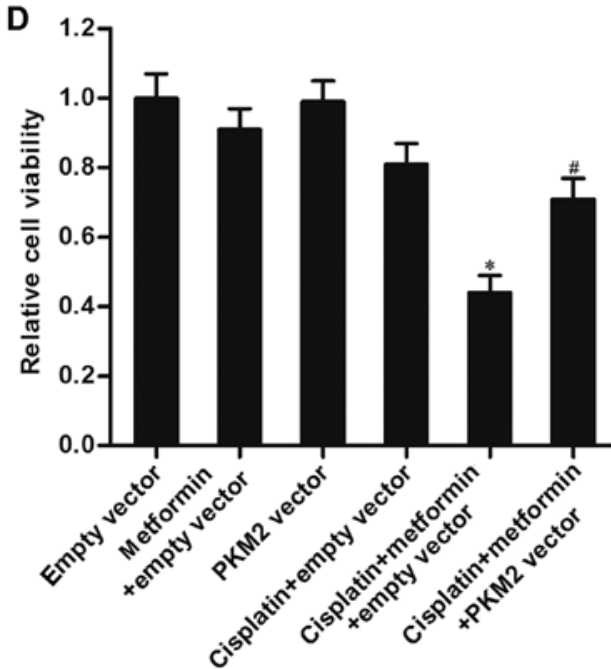

Figure 3. Metformin resensitizes HOS cancer stem cells (CSCs) to cisplatin by downregulating the expression of PKM2. (A) Cell viability of HOS CSCs was measured by CCK-8 assays after they were treated with cisplatin $(8 \mu \mathrm{M})$ and metformin $(2 \mathrm{mM})$. ${ }^{*} \mathrm{P}<0.05$ vs. cisplatin group. (B) IC 50 of cisplatin to $\mathrm{HOS}$ CSCs was determined according to the results of CCK- 8 assays. ${ }^{*} \mathrm{P}<0.05$ vs. control group. (C) Western blot analysis was performed to evaluate the effect of metformin $(2 \mathrm{mM})$, cisplatin $(8 \mu \mathrm{M})$ and PKM2 vector on changing the expression of PKM2. (D) PKM2 vector abolished the synergistic effect of metformin $(2 \mathrm{mM})$ on cisplatin $(8 \mu \mathrm{M})$-induced cell death. ${ }^{*} \mathrm{P}<0.05$ vs. cisplatin + empty vector group. ${ }^{*} \mathrm{P}<0.05$ vs. cisplatin + metformin + empty vector group.

CSCs. However, metformin treatment significantly decreased the consumption of glucose as well as increased the production of lactate in HOS CSCs. In addition, transfection with PKM2 vector restored the glucose uptake and lactate production in HOS CSCs treated with metformin. These results demonstrated that metformin had the ability to weaken the glucose metabolism by inhibiting the expression of PKM2. Furthermore, as the results of glucose metabolism reduction, metformin decreased the production of ATP in HOS CSCs (Fig. 5C).

Combination with metformin and cisplatin induce proliferation inhibition and apoptosis in HOS CSCs. In tumor cells, glucose metabolism and intracellular ATP regulate cell proliferation and chemoresistance $(22,23)$. In our study, we found 


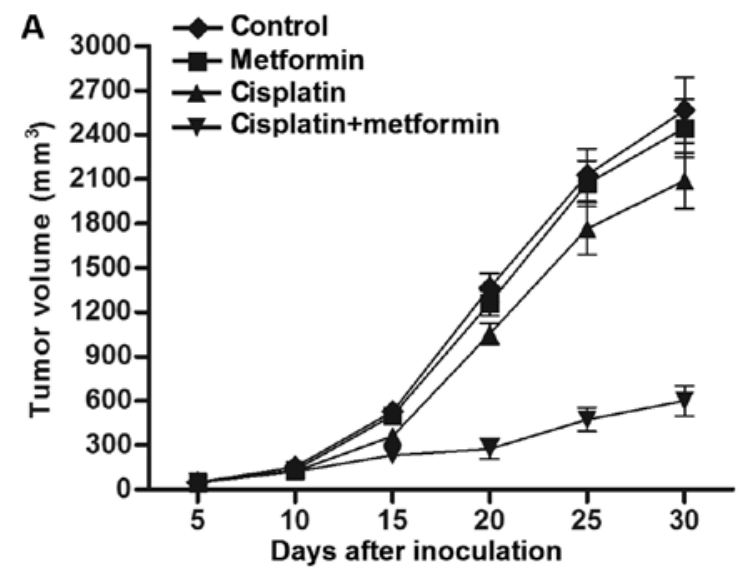

B

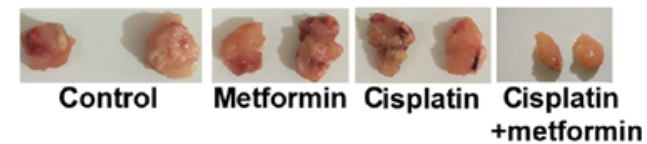

C

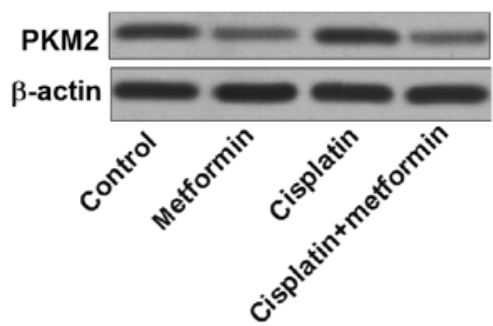

Figure 4. Metformin resensitizes HOS cancer stem cells (CSCs) to cisplatin in vivo. (A) Antitumor effect of cisplatin and metformin on HOS CSCs in vivo model. (B) Effect of cisplatin and metformin on changing the expression of PKM2 in tumor tissues formatted by HOS CSCs.
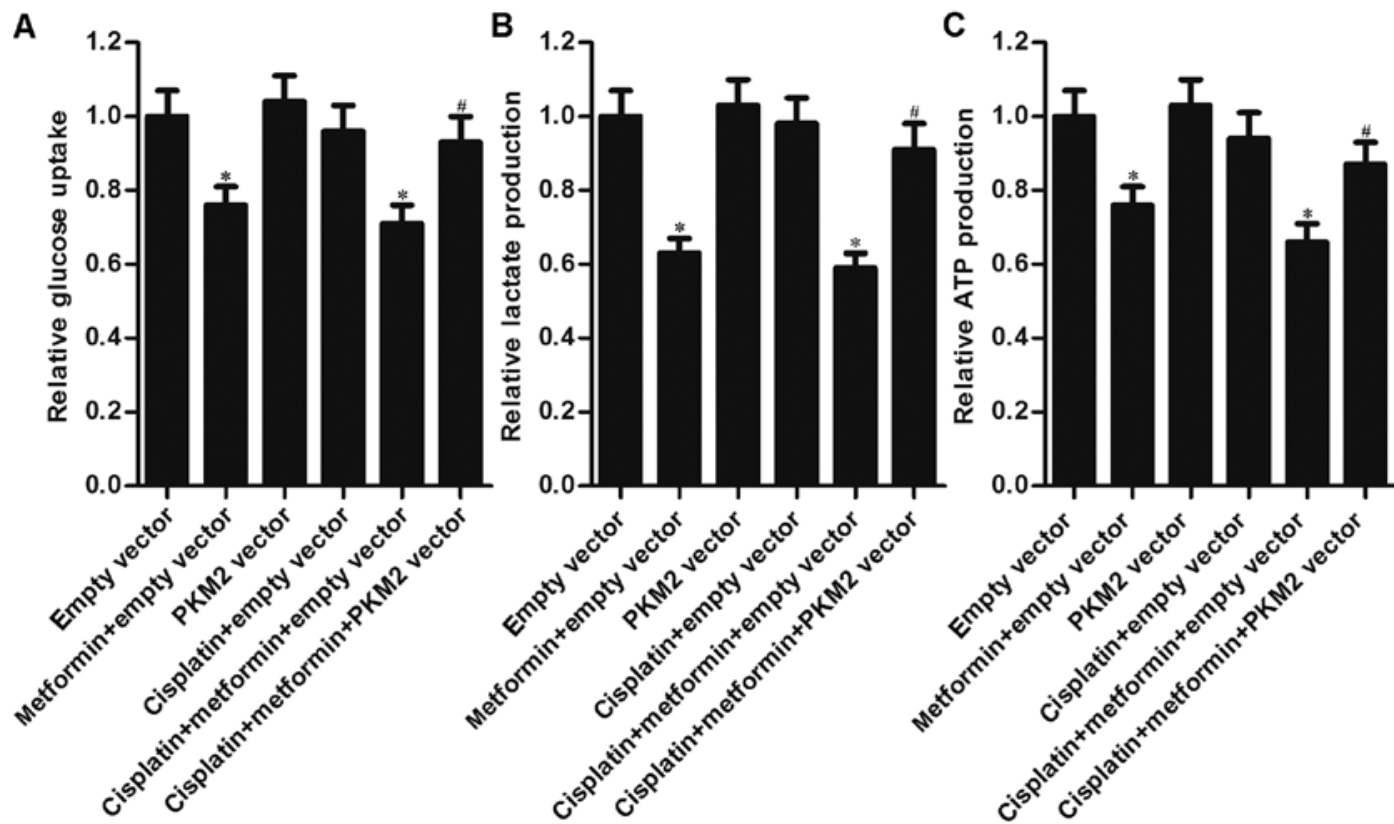

Figure 5. Metformin weakens the glucose metabolism in HOS cancer stem cells (CSCs). (A) Effect of metformin ( $2 \mathrm{mM})$ and cisplatin $(8 \mu \mathrm{M})$ on the consumption of glucose in HOS CSCs. " $\mathrm{P}<0.05$ vs. empty vector group. ${ }^{*} \mathrm{P}<0.05$ vs. cisplatin + metformin + empty vector group. (B) Effect of metformin $(2 \mathrm{mM})$ and cisplatin $(8 \mu \mathrm{M})$ on the production of lactate in HOS CSCs. ${ }^{\mathrm{P}}<0.05$ vs. empty vector group. ${ }^{*} \mathrm{P}<0.05$ vs. cisplatin + metformin + empty vector group. (C) Effect of metformin $(2 \mathrm{mM})$ and cisplatin $(8 \mu \mathrm{M})$ on the production of ATP in HOS CSCs. ${ }^{*} \mathrm{P}<0.05$ vs. empty vector group. ${ }^{\prime \prime} \mathrm{P}<0.05$ vs. cisplatin + metformin + empty vector group.

that combination with metformin and cisplatin induced drastic proliferation inhibition in HOS CSCs. However, enforced expression of PKM2 restored the proliferation of HOS CSCs which were co-treated with metformin and cisplatin (Fig. 6A). Furthermore, we observed that metformin treatment reduced the resistance of HOS CSCs to cisplatin-induced apoptosis. On the other hand, transfection with PKM2 vector impaired the effect of cisplatin plus metformin (Fig. 6B and C). Taken together, we demonstrated that metformin promoted cisplatininduced proliferation inhibition and apoptosis in HOS CSCs by downregulating the expression of PKM2.

Effect of metformin on multidrug resistance in OS stem cells. Multidrug resistance is a major obstacle for chemotherapy of OS. We therefore treated OS stem cells (HOS CSCs, Saos-2 CSCs and MG-63 CSCs) with three kinds of chemotherapeutic drugs (cisplatin, doxorubicin and 5-fluorouracil). As shown in Fig. 7A, co-treatment with metformin significantly increased the sensitivity of HOS CSCs, Saos-2 CSCs and MG-63 CSCs to cisplatin, doxorubicin and 5-fluorouracil. Metformin treatment obviously decreased the $\mathrm{IC}_{50}$ of cisplatin, doxorubicin and 5-fluorouracil to HOS, Saos-2 and MG-63 OS stem cells (Fig. 7B). We demonstrated that metformin can increase the sensitivity of OS stem cells to chemotherapy.

\section{Discussion}

Cisplatin is a cytotoxic drug that is used for treatment of OS (24,25). Mechanism by which cisplatin kills tumor cells is dependent on the formation of cross-linking DNA by interacting with it. When the DNA repair mechanism in tumor fails to neutralize the DNA damage induced by cisplatin, apoptosis 


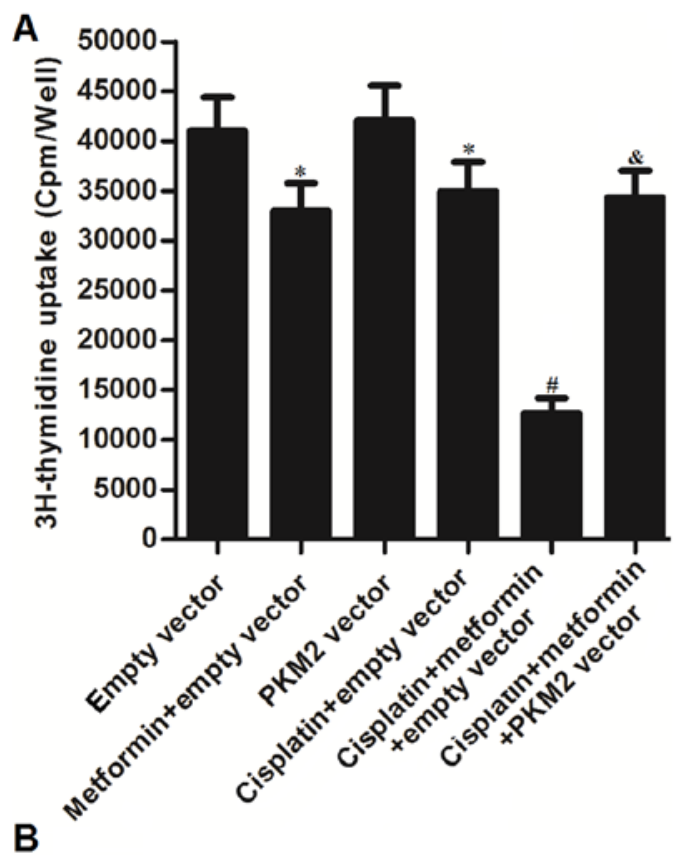

C
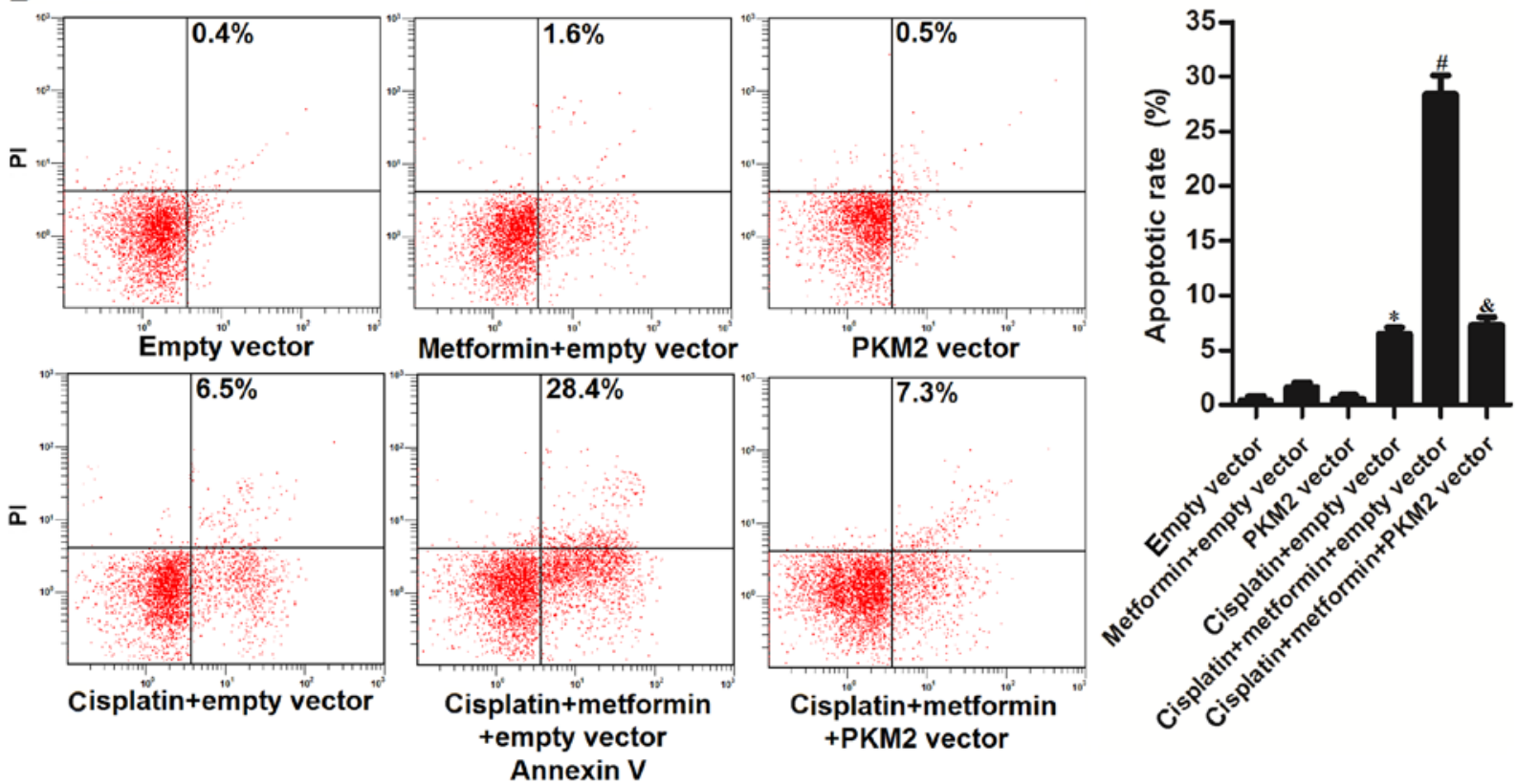

Figure 6. Metformin promoted cisplatin-induced proliferation inhibition and apoptosis in HOS. (A) ${ }^{3} \mathrm{H}$-thymidine incorporation assays were performed to detect the proliferation of HOS cancer stem cells (CSCs) treated with metformin $(2 \mathrm{mM})$, cisplatin $(8 \mu \mathrm{M})$ and PKM2 vector. ${ }^{*} \mathrm{P}<0.05$ vs. empty vector group. ${ }^{\text {"}} \mathrm{P}<0.05$ vs. cisplatin + empty vector group. ${ }^{\circledR} \mathrm{P}<0.05$ vs. cisplatin + metformin + empty vector group. (B) Flow cytometry analysis was performed to measure apoptosis induced by metformin $(2 \mathrm{mM})$, cisplatin $(8 \mu \mathrm{M})$ and PKM2 vector. (C) Western blot analysis was performed to detect the cleavage of caspase-9 and caspase-3 in HOS CSCs treated with metformin $(2 \mathrm{mM})$, cisplatin $(8 \mu \mathrm{M})$ and PKM2 vector.

occurs $(26,27)$. However, chemotherapy resistance often occurs in OS due to the repeated use of cisplatin (28). Recent studies demonstrate that one important factor in tumor recurrence is the existence of cancer stem cells (CSCs) which are resistant to chemotherapy $(29,30)$. Similarly in the present study, HOS, Saos-2 and MG-63 CSCs exhibited significant resistance to cisplatin compared with their corresponding non-CSCs. We proved that OS stem cells showed high $\mathrm{IC}_{50}$ of cisplatin. In view of the high proliferation and oncogenicity of stem cells, increasing sensitivity of OS stem cells to chemotherapy may represent an effective strategy against treatment failure.

Pyruvate kinase isoenzyme M2 (PKM2) in cancer cells catalyzes the final step reaction of glycolysis by converting phosphoenolpyruvate (PEP) and ADP to pyruvate and ATP. Therefore PKM2 is a key regulator of glucose metabolism in tumor cells (31). Recent studies indicate that overexpression of PKM2 is associated with chemoresistance. PKM2 inhibition can restore sensitivity of colon cancer cells, liver cancer cells and multiple myeloma cells to chemotherapy $(20,32,33)$. In our study, we observed significant overexpression of PKM2 in OS stem cells rather than the non-CSCs. As PKM2 siRNA enhanced the cisplatin-induced cell death of HOS, Saos-2 and MG-63 CSCs, we proved that knockdown of PKM2 can restore the sensitivity of OS stem cells to cisplatin treatment. Metformin has been reported to show biological effects of reducing the occurrence of some cancers, and the mecha- 

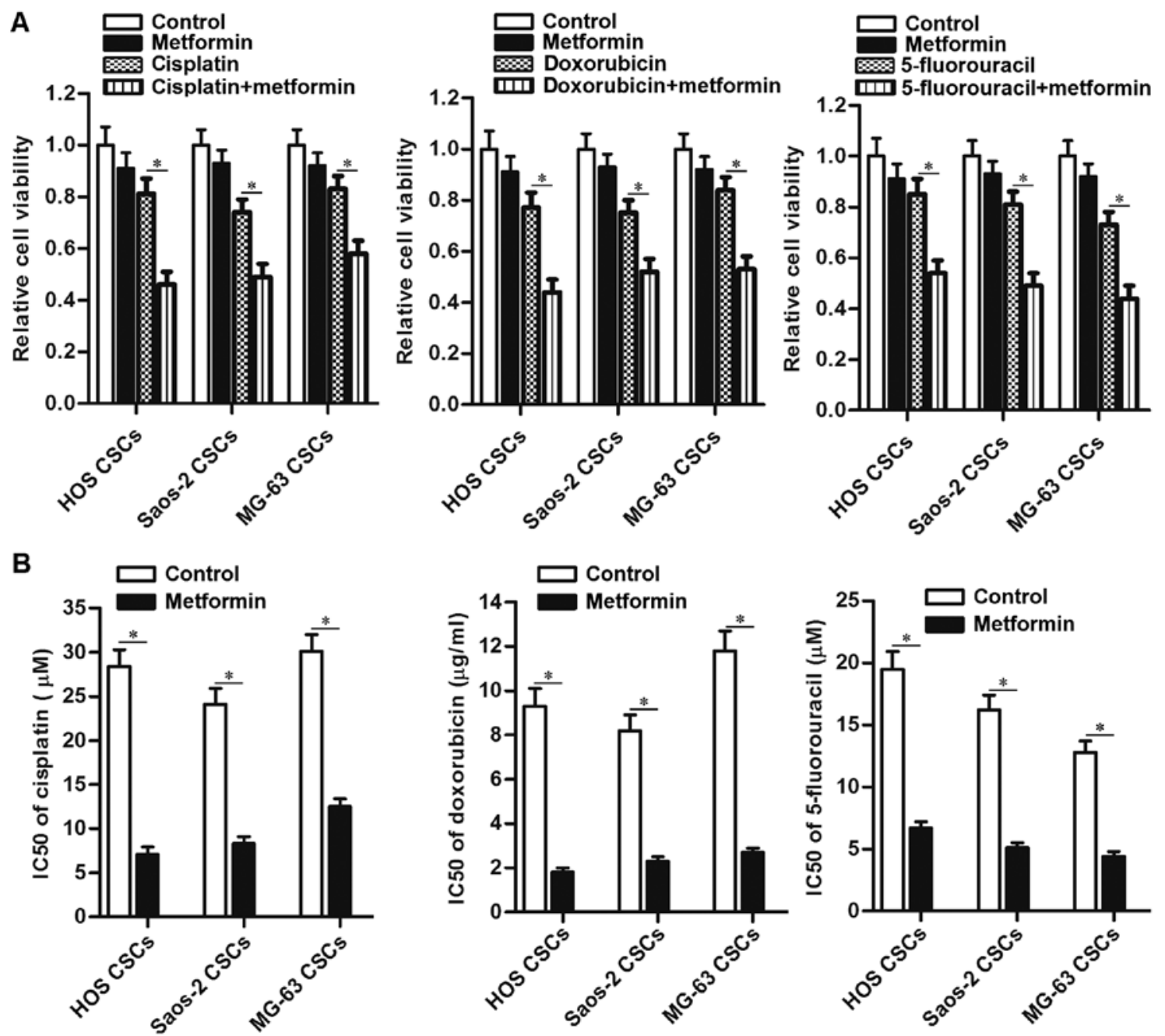

Figure 7. Effect of metformin on multidrug resistance in OS stem cells. (A) HOS, Saos-2 and MG-63 CSCs were treated with metformin (2 mM), cisplatin $(8 \mu \mathrm{M})$, doxorubicin $(2 \mu \mathrm{g} / \mathrm{ml})$ and 5-fluorouracil $(5 \mu \mathrm{M})$ for $48 \mathrm{~h}$. CCK-8 assays were performed to detect the sensitivity of OS stem cells to these chemotherapeutic drugs. ${ }^{*} \mathrm{P}<0.05$. (B) Comparison of $\mathrm{IC}_{50}$ for cisplatin, doxorubicin and 5-fluorouracil to OS stem cells between control group and metformin group. ${ }^{*} \mathrm{P}<0.05$.

nism is associated with the inhibition of PKM2 induced by metformin (34). Consistent with this, our results proved that metformin can decrease the expression of PKM2 in OS stem cells. Sensitization of metformin to cisplatin-induced cell death is dependent on the inhibition of PKM2.

PKM2 regulates glucose metabolism and ATP production in tumor cells (31). Previous research has demonstrated that intracellular ATP levels are the switch to chemoresistance. Bioprocesses of drug efflux, drug inactivation and DNA damage repair require the consumption of ATP. Therefore low-levels of ATP reduce the resistance of drug-induced apoptosis $(23,35)$.

Herein, we demonstrated that metformin treatment reduced the production of ATP in OS stem cells by inhibiting the PKM2 expression. Subsequently, metformin significantly sensitized the OS stem cells to cisplatin-induced apoptosis and activation of caspase- 9 and caspase-3. Furthermore, resistance of OS stem cells to some other chemotherapeutic agents such as doxorubicin and 5-fluorouracil was also inhibited by metformin co-treatment. It is proved that metformin/PKM2 axis is associated with chemosensitivity in OS stem cells.

In conclusion, we provide strong evidence that metformin mediates chemosensitivity in OS stem cells by targeting PKM2.
These findings suggest that combination with metformin and chemotherapeutic agents is a promising strategy for treatment of OS.

\section{Acknowledgements}

Thanks are due to all the contributors who assisted with this study.

\section{References}

1. Siegel R, Naishadham D and Jemal A: Cancer statistics, 2013. CA Cancer J Clin 63: 11-30, 2013.

2. Huang J, Liu K, Song D, Ding M, Wang J, Jin Q and Ni J: Krüppel-like factor 4 promotes high-mobility group box 1-induced chemotherapy resistance in osteosarcoma cells. Cancer Sci 107: 242-249, 2016.

3. Sun Y, He N, Dong Y and Jiang C: MiR-24-BIM-Smac/DIABLO axis controls the sensitivity to doxorubicin treatment in osteosarcoma. Sci Rep 6: 34238, 2016.

4. Shackleton M, Quintana E, Fearon ER and Morrison SJ: Heterogeneity in cancer: Cancer stem cells versus clonal evolution. Cell 138: 822-829, 2009.

5. Clarke MF and Fuller M: Stem cells and cancer: Two faces of eve. Cell 124: 1111-1115, 2006. 
6. Martins-Neves SR, Paiva-Oliveira DI, Wijers-Koster PM, Abrunhosa AJ, Fontes-Ribeiro C, Bovée JV, Cleton-Jansen AM and Gomes CM: Chemotherapy induces stemness in osteosarcoma cells through activation of $\mathrm{Wnt} / \beta$-catenin signaling. Cancer Lett 370: 286-295, 2016.

7. Yang J, Guo W, Wang L, Yu L, Mei H, Fang S, Ji P, Liu Y, Liu G and Song Q: Cisplatin-resistant osteosarcoma cells possess cancer stem cell properties in a mouse model. Oncol Lett 12 : 2599-2605, 2016.

8. Gonçalves C, Martins-Neves SR, Paiva-Oliveira D, Oliveira VE, Fontes-Ribeiro C and Gomes CM: Sensitizing osteosarcoma stem cells to doxorubicin-induced apoptosis through retention of doxorubicin and modulation of apoptotic-related proteins. Life Sci 130: 47-56, 2015.

9. Ni M, Xiong M, Zhang X, Cai G, Chen H, Zeng Q and Yu Z: Poly(lactic-co-glycolic acid) nanoparticles conjugated with CD133 aptamers for targeted salinomycin delivery to CD133 osteosarcoma cancer stem cells. Int J Nanomedicine 10: $2537-2554,2015$

10. Feng X, Jiang J, Shi S, Xie H, Zhou L and Zheng S: Knockdown of miR-25 increases the sensitivity of liver cancer stem cells to TRAIL-induced apoptosis via PTEN/PI3K/Akt/Bad signaling pathway. Int J Oncol 49: 2600-2610, 2016.

11. Chen Y, Zhang F, Tsai Y, Yang X, Yang L, Duan S, Wang X Keng P and Lee SO: IL-6 signaling promotes DNA repair and prevents apoptosis in $\mathrm{CD} 133^{+}$stem-like cells of lung cancer after radiation. Radiat Oncol 10: 227, 2015.

12. Adler AI, Shaw EJ, Stokes T and Ruiz F; Guideline Development Group: Newer agents for blood glucose control in type 2 diabetes: Summary of NICE guidance. BMJ 338: b1668, 2009.

13. Tseng CH: Metformin reduces thyroid cancer risk in Taiwanese patients with type 2 diabetes. PLoS One 9: e109852, 2014.

14. Dowling RJ, Niraula S, Stambolic V and Goodwin PJ: Metformin in cancer: Translational challenges. J Mol Endocrinol 48: R31-R43, 2012

15. Zhao W, Zhang $\mathrm{X}$, Liu J, Sun B, Tang $\mathrm{H}$ and Zhang $\mathrm{H}$ miR-27a-mediated antiproliferative effects of metformin on the breast cancer cell line MCF-7. Oncol Rep 36: 3691-3699, 2016.

16. Franciosi M, Lucisano G, Lapice E, Strippoli GF, Pellegrini F and Nicolucci A: Metformin therapy and risk of cancer in patients with type 2 diabetes: Systematic review. PLoS One 8: e71583, 2013

17. El-Mir MY, Nogueira V, Fontaine E, Avéret N, Rigoulet M and Leverve X: Dimethylbiguanide inhibits cell respiration via an indirect effect targeted on the respiratory chain complex I. J Biol Chem 275: 223-228, 2000.

18. Silvestri A, Palumbo F, Rasi I, Posca D, Pavlidou T, Paoluzi S, Castagnoli L and Cesareni G: Metformin induces apoptosis and downregulates pyruvate kinase M2 in breast cancer cells only when grown in nutrient-poor conditions. PLoS One 10 e0136250, 2015

19. Yang W and Lu Z: Regulation and function of pyruvate kinase M2 in cancer. Cancer Lett 339: 153-158, 2013.
20. He Y, Wang Y, Liu H, Xu X, He S, Tang J, Huang Y, Miao X, Wu Y, Wang Q, et al: Pyruvate kinase isoform M2 (PKM2) participates in multiple myeloma cell proliferation, adhesion and chemoresistance. Leuk Res 39: 1428-1436, 2015.

21. Dayton TL, Jacks T and Vander Heiden MG: PKM2, cancer metabolism, and the road ahead. EMBO Rep 17: 1721-1730, 2016.

22. Garber K: Energy deregulation: Licensing tumors to grow. Science 312: 1158-1159, 2006.

23. Zhou Y, Tozzi F, Chen J, Fan F, Xia L, Wang J, Gao G, Zhang A, $\mathrm{Xia} \mathrm{X}$, Brasher $\mathrm{H}$, et al: Intracellular ATP levels are a pivotal determinant of chemoresistance in colon cancer cells. Cancer Res 72: 304-314, 2012.

24. Ren XF, Mu LP, Jiang YS, Wang L and Ma JF: LY2109761 inhibits metastasis and enhances chemosensitivity in osteosarcoma MG-63 cells. Eur Rev Med Pharmacol Sci 19: 1182-1190, 2015.

25. Yang Q, Zhang S, Kang M, Dong R and Zhao J: Synergistic growth inhibition by sorafenib and cisplatin in human osteosarcoma cells. Oncol Rep 33: 2537-2544, 2015.

26. Davis W Jr, Ronai Z and Tew KD: Cellular thiols and reactive oxygen species in drug-induced apoptosis. J Pharmacol Exp Ther 296: 1-6, 2001.

27. Casares C, Ramírez-Camacho R, Trinidad A, Roldán A, Jorge E and García-Berrocal JR: Reactive oxygen species in apoptosis induced by cisplatin: Review of physiopathological mechanisms in animal models. Eur Arch Otorhinolaryngol 269: 2455-2459, 2012.

28. Gao JZ, Chen FH, Wang L, Wei H and Meng SL: YM155 inhibits tumor growth and enhances chemosensitivity to cisplatin in osteosarcoma. Eur Rev Med Pharmacol Sci 19: 2062-2069, 2015.

29. Liu J, Tang Q, Li S and Yang X: Inhibition of HAX-1 by miR-125a reverses cisplatin resistance in laryngeal cancer stem cells. Oncotarget 7: 86446-86456, 2016.

30. Abarrategi A, Tornin J, Martinez-Cruzado L, Hamilton A, Martinez-Campos E, Rodrigo JP, González MV, Baldini N, Garcia-Castro J and Rodriguez R: Osteosarcoma: Cells-oforigin, cancer stem cells, and targeted therapies. Stem Cells Int 2016: 3631764, 2016

31. Li Z, Yang P and Li Z: The multifaceted regulation and functions of PKM2 in tumor progression. Biochim Biophys Acta 1846: 285-296, 2014

32. He J, Xie G, Tong J, Peng Y, Huang H, Li J, Wang N and Liang H: Overexpression of microRNA-122 re-sensitizes 5-FU-resistant colon cancer cells to 5-FU through the inhibition of PKM2 in vitro and in vivo. Cell Biochem Biophys 70: 1343-1350, 2014.

33. He Y, Wang Y, Liu H, Xu X, He S, Tang J, Huang Y, Miao X, Wu Y, Wang Q, et al: Pyruvate kinase isoform M2 (PKM2) participates in multiple myeloma cell proliferation, adhesion and chemoresistance. Leuk Res 39: 1428-1436, 2015.

34. Chen G, Feng W, Zhang S, Bian K, Yang Y, Fang C, Chen M, Yang $\mathrm{J}$ and Zou X: Metformin inhibits gastric cancer via the inhibition of HIF1 $\alpha /$ PKM2 signaling. Am J Cancer Res 5: 1423-1434, 2015.

35. Longley DB and Johnston PG: Molecular mechanisms of drug resistance. J Pathol 205: 275-292, 2005. 